\title{
Outcome of Patients with ST-T Changes in Non ST- Segment Elevation Myocardial Infarction
}

\author{
Mohammad Arifur Rahman, ${ }^{1 *}$ Md. Shahimur Parvez, ${ }^{2}$ Arifin Islam Lita ${ }^{3}$ \\ ${ }^{1}$ Consultant (Cardiology), Sheikh Fazilatunnessa Mujib Memorial KPJ Specialized Hospital, Bangladesh \\ ${ }^{2}$ Assistant Professor, Department of Cardiology, Enam Medical College \& Hospital, Bangladesh \\ ${ }^{3}$ Consultant (Covid), Department of Medicine, Holy Family Red Crescent Medical College \& Hospital, Bangladesh
}

\begin{abstract}
Introduction: The myocardial infarction (MI) mortality has decreased markedly during recent decades, a decrease that has multiple causes. In spite of improvements, the incidence of acute MI has remained high and cardiovascular disease is still the leading cause of death, afflicting almost $50 \%$ of both men and women. Objective: To assess in hospital Outcome of Patients with ST-T Changes In Non ST-Segment Elevation Myocardial Infarction.
\end{abstract}

Methods: A hospital-based cross-sectional study. It was done on one year duration (January 2019-December 2019) in the coronary care unit of Department of Cardiology, Sheikh Fazilatunnessa Mujib Memorial KPJ Specialized Hospital, Gazipur, Bangladesh. Study population were patients attending at hospital having Non ST-elevation MI screened out by clinical examination and electro-cardiography (ECG) and biochemical marker (S.Troponin I). Considering the inclusion and exclusion criteria a total number of 115 patients presented with NSTEMI were included in the study.

Results: The 115 Non ST Elevated patients were included in the study comprising 50 women and 65 men. The study population consisted of 51 patients ( 16 women, 35 men) aged under 50 years and 64 patients ( 34 women, 30 men) aged 50 years or older. The mean age of NSTEMI in study population is 52.45 years and the mean age of women in the study is 55.70 years and the mean age of men in the study is 49.49 years.

Conclusion: In the present study men represent a large population of patients with NSTEMI than women. Smoking is the most alarming risk factors in young male while diabetes and hypertension in female. There are substantial differences in baseline characteristics between a male and a female population with NSTEMI. So that definite measures can be formulated to the patients properly of that gender specific group.

Keywords: Non-ST segment elevation myocardial infarction, Gender, ACS

\section{Introduction}

The myocardial infarction (MI) mortality has decreased markedly during recent decades, a decrease that has multiple causes. In spite of improvements, the incidence of acute MI has remained high and cardiovascular disease is still the leading cause of death, afflicting almost $50 \%$ of both men and women. Coronary heart disease accounts for most of the cardiovascular events, and MI is the single most important contributor to the mortality and morbidity. ${ }^{1}$ Historically, fewer women than men have been included in studies on coronary heart disease (CHD). Whether this is caused by lower incidence in women, especially at younger age, or actual exclusion of women from the trials have been debated. ${ }^{2}$ The consequence is that evidence base for several treatments are fewer forms for women than for men. Lack of gender-specific knowledge has emerged as an important issue in the management of non ST-elevation acute coronary syndromes where some data have indicated a difference in benefit from a routine invasive strategy according to gender. ${ }^{3-6}$ There are also reports that women have been managed less intensively, with worse outcome, compared to men. For example, women have less often received reperfusion therapy, early antithrombotic therapy and antiplatelet therapy at discharge., ${ }^{7,8}$ Moreover, men have more often been referred for coronary angiography. There are several important differences in background
Quick Response Code:

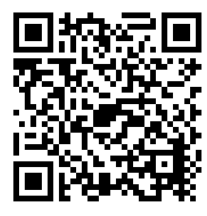

*Corresponding author: Mohammad Arifur Rahman, Consultant (Cardiology), Sheikh Fazila-
tunnessaMujib Memorial KPJ Specialized Hospital, Gazipur, Bangladesh
Received: 14 May, $2021 \quad$ Published: 01 June, 2021
Citation: Mohammad AR, Shahimur P, Arifin IL. Outcome of Patients with ST-T Changes in
Non ST-Segment Elevation Myocardial Infarction. Curr Inv Cln Med Res. 2021;1(1):1-4. DOI:
10.53902/CICMR.2021.01.000504


characteristics between a female and a male population with acute coronary syndromes (ACS); for example, females are older and have more co-morbid conditions. ${ }^{9}$ Today, ACS is the leading cause of death in both gender of the western world and during the last two decades there has been an ongoing debate about women and ACS and whether women and men suffering from this syndrome differ in baseline characteristics, clinical presentation, treatment and outcome. ${ }^{10}$ Early mortality among patients hospitalized with acute MI has been systematically rumored to be higher among ladies than men. FRISC II and RITA three trials rumored worsening outcomes among ladies with ACS who were treated invasively. This finding has raised doubt on whether or not treatment in ladies and men ought to be similar. ${ }^{11}$ Alternative studies work age-sex variations in short mortality on the far side the hospital keep support higher semi-permanent mortality rates among ladies, significantly younger ladies, compared with men at same ages. Vaccarino et al discovered that girls younger than sixty years old-time had the next morbidity than men and therefore the mortality risk for ladies compared with men shrunken with increasing age, to the purpose wherever ladies within the oldest age showed a lower two year morbidity than men of comparable age. ${ }^{12,13}$ Studies examination management and outcome in men and ladies area unit, for obvious reasons, not randomised why truthful comparisons have confidence applied mathematics strategies to regulate for discovered variations in background characteristics. ${ }^{14,15}$ to make your mind up whether or not its gender in and of itself or alternative characteristics that account for discovered variations in management and outcome between the genders, giant study populations, with data on potential confounders, area unit required to perform correct changes to boost the individual management of NSTEMI patients its vital to clarify if we have a tendency to, in real world clinical apply, treat ladies otherwise than men. It's additionally vital to guage if there are unit variations in result of treatments between the genders, and if discovered variations area unit thanks to gender analyze. ${ }^{16,17}$

\section{Methodology}

A hospital-based cross-sectional study. It was done on one year duration (January 2019-December 2019) in the coronary care unit (CCU) of Department of Cardiology, Sheikh Fazilatunnessa Mujib Memorial KPJ Specialized Hospital, Gazipur, Bangladesh. Study population were patients attending at hospital having Non ST-elevation MI screened out by clinical examination and electro-cardiography (ECG) and biochemical marker (S. Troponin I). Considering the inclusion and exclusion criteria a total number of 115 patients presented with NSTEMI were included in the study. After collection all the data were checked and edited. Then data were entered into computer with the help of software SPSS for Windows programmed version 19.0. After frequency run, data were cleaned and frequencies were checked. An analysis plan was developed keeping in view with the objectives of the study.

\section{Results}

The 115 Non ST Elevated patients were included in the study comprising 50 women and 65 men. The study population consisted of 51 patients ( 16 women, 35 men) aged under 50 years and 64 patients ( 34 women, 30 men) aged 50 years or older. The mean age of NSTEMI in study population is $\mathbf{5 2 . 4 5}$ years and the mean age of women in the study is 55.70 years and the mean age of men in the study is 49.49 years. Commonly ST depression and T wave inversion are significantly seen in women. Other ECG changes include atrial fibrillation, LBBB or RBBB pattern but all of these changes have no significance differences in relation to sex and diabetes than men, and they were significantly less frequent in current smokers. Older women $>50$ years more often were hypertensive, while men more often had a history of smoking. A part from smoking, the frequency of cardiovascular risk factors was similar in both female age groups, while the incidence of arterial hypertension, diabetes was higher in older than in younger men. Regarding in hospital outcome there is persistent chest pain in 23(46\%) women and $16(24.6 \%)$ men heart failure is present in $3(6 \%)$ women and $2(3.1 \%)$ men. The differences in incidence of persistent chest pain and heart failure are statistically significant between the two genders (Tables 1\&2) (Figure 1).

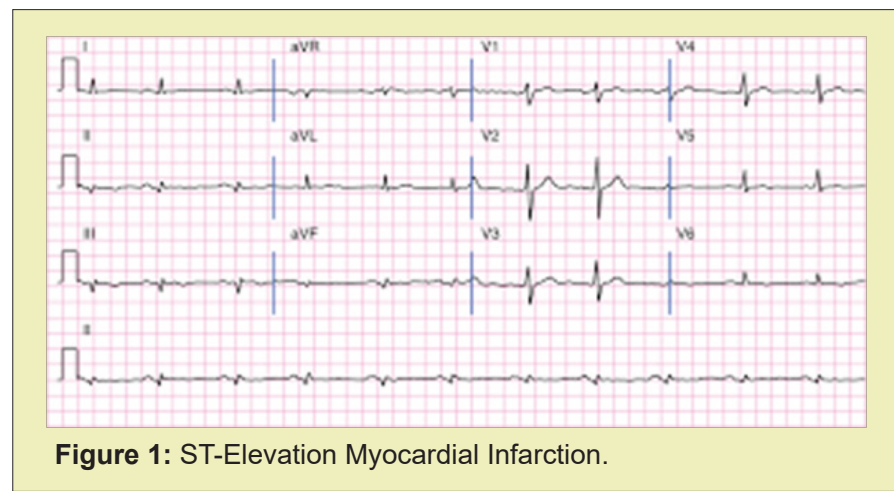

Table 1: Distribution of risk factors according to sex $(n=115)$.

\begin{tabular}{|c|c|c|c|c|c|c|}
\hline \multirow{2}{*}{ Risk factors } & \multicolumn{2}{|c|}{$<50$ years } & \multirow{2}{*}{$p$ value } & \multicolumn{2}{|c|}{$\geq 50$ years } & \multirow{2}{*}{$p$ value } \\
\hline & Male N=35 & Female $\mathrm{N}=16$ & & Male $\mathrm{N}=30$ & Female $N=34$ & \\
\hline HTN & $12(34.3)$ & $11(68.7)$ & 0.02 & $15(50)$ & $27(79.4)$ & 0.01 \\
\hline DM & $06(17.1)$ & $09(56.2)$ & 0.007 & $14(46.7)$ & $11(32.4)$ & 0.24 \\
\hline CKD & - & - & - & $02(6.7)$ & $02(5.9)$ & 1.0 \\
\hline Dyslipidemia & $12(34.3)$ & 03(18.7) & 0.33 & $05(16.7)$ & $03(8.8)$ & 0.45 \\
\hline Smoking & $27(77.1)$ & $01(6.3)$ & $<0.001$ & $16(53.3)$ & $05(14.7)$ & 0.001 \\
\hline $\begin{array}{l}\text { Coronary Heart } \\
\text { Disease }\end{array}$ & $07(20.0)$ & $06(37.5)$ & 0.18 & $04(13.3)$ & $08(23.5)$ & 0.29 \\
\hline
\end{tabular}

HTN: Hypertension; DM: Diabetes Mellitus; CKD:Chronic kidney disease 
Table 2: In hospital outcome according to distribution of gender $(n=115)$.

\begin{tabular}{|c|c|c|c|c|}
\hline \multirow{2}{*}{ Outcome } & \multicolumn{2}{|c|}{ Sex } & \multirow{2}{*}{ Total } \\
\cline { 2 - 4 } & Male (n=65) & Female (n=50) & 39 & \multirow{2}{*}{0.030} \\
\hline Persistent chest pain & 16 & 23 & 5 & 0.035 \\
\hline Heart failure & 2 & 3 & 5 & 0.34 \\
\hline Cardiogenic shock & 2 & 3 & 2 & 0.043 \\
\hline Arrhythmia & 1 & 1 & 3 & 0.49 \\
\hline Reinfarction & 2 & 1 & 3 & 0.49 \\
\hline Major bleeding & 2 & 1 & 3 & 0.49 \\
\hline CVD/Stroke/TIA & 2 & 1 & 3 & 0.49 \\
\hline Death & 2 & 1 & & 3 \\
\hline
\end{tabular}

CVD:Cerebro-Vascular Disease; TIA:Transient Ischemic Attack

\section{Discussion}

The debate on the reasons for the differences in mortality and morbidity between women and men with NSTEMI is still ongoing and no full agreement has been reached so far. Many researchers associate poorer outcomes in women with co-morbidities, clinical manifestation and adverse events.

\section{Risk factors and baseline characteristics}

In the present study, there were more men in the age group of $<50$ years, while in the older group the percentage of men and women were practically the same. Women tend to live longer and develop cardiovascular disease at a later age, which means that both in younger and older age groups women are older than men and they have additional risk factors. In the general population of patients with NSTEMI, there is a discrepancy in the prevalence of conventional risk factors (arterial hypertension, diabetes, dyslipidemia, smoking, obesity, prior MI) between sexes. Women $<50$ years tend to have hypertension, diabetes and family history of coronary heart diseases more often, while smoking and dyslipidemia are more frequent in men. The incidence of hypertension increases with age in both sexes. It seems that the frequency of diabetes mellitus is more in women than men of age $<50$ years but it is reverse in age $>50$ years patients. There is no significant differences in women and men regarding chronic kidney disease (CKD), dyslipidemia and family history of coronary heart disease.

\section{Electrocardiographic changes}

A negative prognostic value of ST-segment depression and T-wave inversion in the index ECG is well established. In our study, there was significant difference in ST-segment depression and $\mathrm{T}$ -wave inversion between men and women $<50$ years age groups; however, atrial fibrillation was observed more frequently in older men $>50$ years.

\section{Echocardiographic changes}

In this study we have found that women $<50$ years have significant echocardiographic changes. There were only significant wall motion abnormalities in case of inferior and lateral wall which was more in female patients. The mean ejection fraction was found more in men $(E F=57.97 \%)$ than women $(E F=55.48 \%)$. But it was not significant. Regarding diastolic dysfunction there was no significant difference in women and men.

\section{In-hospital management}

In our study we have found that women were more likely to be treated with beta-blockers, angiotensin converting enzyme inhibitor (ACEi) and diuretics which may reflect the higher rate of hypertension and heart failure. After age adjustment there was no difference between the gender in treatment with heparin/low molecular weight heparin (LMWH), statins, nitrates were used infrequently in both men and women in our data, but less often in women.

\section{Complications}

In our observation $24.6 \%$ men experienced persistent chest pain in comparison to $46 \%$ women and it was statistically significant. The incidence of heart failure increases with age and reaches $12.5 \%$ in patients younger than 65 years and $22 \%$ to $41 \%$ in patients over 65 years. In many registries, and also in our study, no differences between young men and women were observed, while women had developed heart failure $(6.0 \%)$ more than men $(3.1 \%)$ and it is statistically significant. Another important finding was arrhythmia, statistically significant in men (20\%) than women (8.0\%). Other complications like cardiogenic shock, re-infarction, cerebro-vascular events were not found to differ significantly between the groups.

\section{Mortality}

In our study we've found that in hospital death occurred solely in two men $(3.1 \%)$ and one women $(2 \%)$ and also the distinction wasn't vital.

\section{Conclusion}

In the present study men represent a large population of patients with NSTEMI than women. Smoking is the most alarming risk factors in young male while diabetes and hypertension in young female. There are substantial differences in baseline characteristics between a male and a female population with NSTEMI. So that definite measures can be formulated to the patients properly of that gender specific group. Female show worse prognosis relating 
to failure and chronic pain. Risk factors identification, early managementare crucial within the primary and secondary interference in young patient with CAD. Adoption and application of latest data relating to sex variations can hopefully cause improve outcomes.

\section{Acknowledgments}

None.

\section{Funding}

None.

\section{Conflicts of Interest}

Author declares that there is no conflict of interest.

\section{References}

1. Maynard C, Every NR, Martin JS, et al. Association of gender and survival in patients with acute myocardial infarction. Arch Intern Med. 1997;157(12):1379-1384

2. Barrett-Connor E. Sex differences in coronary heart disease. Why are women so superior? The 1995 Ancel Keys Lecture. Circulation. 1997;95(1):252-264.

3. Wingard DL, Cohn BA, Kaplan GA, et al. Sex differentials in morbidity and mortality risks examined by age and cause in the same cohort. Am J Epidemiol. 1989;130(3):601-610.

4. Ahmed S, Cannon CP, Giugliano RP, et al. The independent and combined risk of diabetes and non endstage renal impairment in non ST segment elevation acute coronary syndromes. Int J Cardiol. 2008;131:105-112.

5. Gilat D, Goldbourt U, Reicher Reiss $\mathrm{H}$, et al. Prognosis of acute myocardial infarction in the elderly. SPRINT Study Group. Harefuah. 1993;124(10):601-668.

6. Avezum A, Makdisse M, Spencer F, et al. Impact of age on management and outcome of acute coronary syndrome: observations from the Global Registry of Acute Coronary Events (GRACE). Am Heart J. 2005;149(1):67-73.

7. Mehta RH, Rathore SS, Radford MJ, et al. Acute myocardial infarction in the elderly: differences by age. J Am Coll Cardiol. 2001;38(3):736-741.

8. Goldberg RJ, McCormick D, Gurwitz JH, et al. Age-related trends in short-and long-term survival after acute myocardial infarction: a 20-year population-based perspective (1975-1995). Am J Cardiol. 1998;82(11):1311-1317.
9. Yan RT, Yan AT, Tan M, et al. Age-related differences in the management and out-come of patients with acute coronary syndromes. Am Heart J. 2006;151:352-359.

10. Thiemann DR, Coresh J, Schulman SP, et al. Lack of benefit for intravenous thrombolysis in patients with myocardial infarction who are older than 75 years. Circulation. 2000;101(19):2239-2246.

11. Stenestrand U, Wallentin L. Fibrinolytic therapy in patients 75 years and older with ST-segment-elevation myocardial infarc-tion: one-year follow-up of a large prospective cohort. Arch Intern Med. 2003;163(8):965-971.

12. Vaccarino V, Krumholz HM, Yarzebski J, et al. Sex differences in 2-year mortality after hospital discharge for myocardial infarction. Intern Med. 2001;134(3):173-181.

13. Alexander KP, Newby LK, Cannon CP, et al. American Heart Association Council on Clinical Cardiology; Society of Geriatric Cardiology. Acute coronary care in the elderly, part I: Non ST segment elevation acute coronary syndromes: a scientific statement for healthcare professionals from the American Heart Association Council on Clinical Cardiology: in collaboration with the Society of Geriatric Cardiology. Circulation. 2007:115:2549-2569.

14. Anand SS, Islam S, Rosengren A, et al. Risk factors for myocardial infarction in women and men: insights from the INTERHEART study. Eur Heart J. 2008;29(7):932-940.

15. Braunwald E: Unstable angina: An etiologic approach to management. Circulation. 1998;98:2219.

16. Mueller C, Neumann FJ, Perach W, et al. Prognostic value of the admission electrocardiogram in patients with unstable angina/non ST segment elevation myocardial infarction treated with very early revascularization. Am J Med. 2004;117:145-150.

17. Stone PH, Thompson B, Anderson HV, et al. Influence of race, sex, and age on management of unstable angina and non-Q-wave myocardial infarction: The TIMI III registry. JAMA. 1996;275(14):1104-1112. 